\title{
Marked tumor response to crizotinib after 4 years of maintenance pemetrexed in a patient with anaplastic lymphoma kinase-positive non-small-cell lung cancer
}

\author{
MIN YU ${ }^{1 *}$, SHUANG ZHANG ${ }^{2 *}$, MEIJUAN HUANG ${ }^{1,2}$ and YOU LU ${ }^{1,2}$ \\ ${ }^{1}$ Department of Thoracic Oncology; ${ }^{2}$ State Key Laboratory of Biotherapy and Cancer Center, \\ West China Hospital, Sichuan University, Chengdu, Sichuan 610041, P.R. China
}

Received January 15, 2014; Accepted March 27, 2014

DOI: $10.3892 / \mathrm{mco} .2014 .280$

\begin{abstract}
Maintenance therapy with pemetrexed is well tolerated and achieves prolonged progression-free and overall survival in patients with advanced lung adenocarcinoma. The echinoderm microtubule-associated protein-like 4 (EML4)-anaplastic lymphoma kinase (ALK) is a recently identified fusion oncogene that exists in $\sim 5 \%$ of non-small-cell lung cancers (NSCLCs). It was demonstrated that ALK-positive NSCLCs exhibit a high response rate to the ALK inhibitor crizotinib. This is the case report of a patient with NSCLC harboring EML4-ALK rearrangement, who experienced slowly progressive disease within 4 years of maintenance treatment with pemetrexed and later exhibited a marked response to crizotinib. The sustained clinical benefits suggest further investigations on anticancer agent administration.
\end{abstract}

\section{Introduction}

It was reported that in 2013, lung cancer was expected to account for $26 \%$ of all female and $28 \%$ of all male cancer-related deaths in the USA (1). Approximately $85 \%$ of lung cancers are non-small-cell lung cancer (NSCLC) and the majority of patients are diagnosed at an advanced stage. The standard first-line platinum-based chemotherapy has achieved modest improvements in overall survival (2). Pemetrexed is an antifolate antineoplastic agent that exerts its effects by disrupting folate-dependent metabolism. Continued chemotherapeutic treatment prior to disease progression with single-agent pemetrexed was found to improve progression-free and overall

Correspondence to: Dr Meijuan Huang, Department of Thoracic Oncology, West China Hospital, Sichuan University, 37 Guo Xue Xiang, Chengdu, Sichuan 610041, P.R. China

E-mail: shuang-4321-@163.com

*Contributed equally

Key words: anaplastic lymphoma kinase-positive non-small-cell lung cancer, adenocarcinoma, crizotinib, pemetrexed survival in advanced lung cancer $(3,4)$. Crizotinib was approved in 2011 by the Food and Drug Administration for the treatment of anaplastic lymphoma kinase (ALK)-rearranged NSCLC. Crizotinib is an orally active small-molecule inhibitor of ALK and the c-Met receptor tyrosine kinase and belongs to the family of 3-benzyloxy-2-aminopyridines. Recently, a phase 3 clinical trial indicated that, compared to chemotherapy, crizotinib prolonged progression-free survival, increased response rates and improved the quality of life in patients with advanced, previously treated ALK-positive NSCLC $(5,6)$.

In this study, we present the case report of a patient with NSCLC harboring echinoderm microtubule-associated protein-like 4 (EML4)-ALK rearrangement. The patient, following discontinuation of second-line therapy, experienced slowly progressive disease within 4 years of maintenance pemetrexed and later exhibited a favorable response to crizotinib. This case indicates that selected patients may continue to benefit from pemetrexed after disease progression.

\section{Case report}

In May 2007, a 63-year-old man presented with a mildly productive cough. The patient had been initially treated for pneumonia in a community hospital; however, as the symptoms did not improve despite treatment, the patient underwent a chest computed tomography (CT) scan in the Sichuan Provincial Hospital, which revealed multiple nodes in the left lower pulmonary lobe and left pleura (Fig. 1A). Biopsy of the pleural nodes was performed by thoracoscopy and the histological examination confirmed the diagnosis of mucinous adenocarcinoma. Immunohistochemical staining was positive for thyroid transcription factor-1, epithelial membrane antigen and lymphocyte-specific protein tyrosine kinase. The patient had an Eastern Cooperative Oncology Group performance status of 1 and received a gemcitabine and cisplatin regimen for 2 cycles. However, treatment was discontinued due to persistent gastrointestinal upset. The patient then received erlotinib orally for 6 months (150 mg daily) and the tumor response according to the Response Evaluation Criteria in Solid Tumors (RECIST) guidelines was stable disease. However, the treatment was discontinued due to erlotinib-associated pulmonary toxicity (interstitial pneumonia). 
A Diagnosis
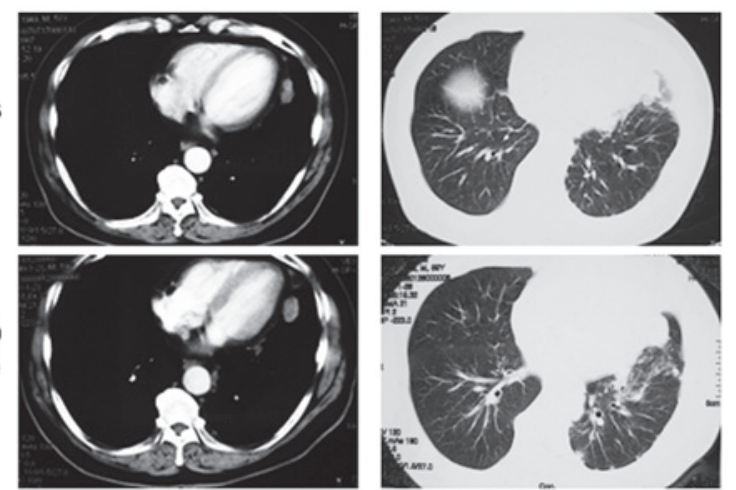

C Before AO
schedule
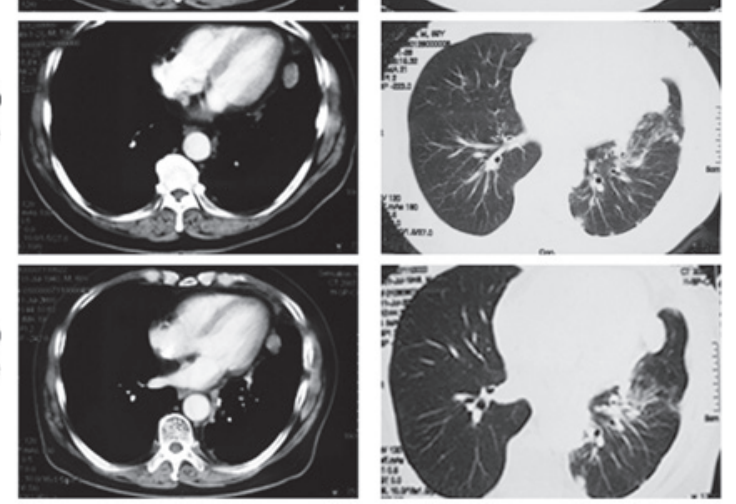

Figure 1. (A) Chest CT at initial presentation. Chest CT (B) before and (C) after pemetrexed and oxaliplatin chemotherapy (AO schedule). Left, mediastinal window. Right, lung window. CT, computed tomography.

A
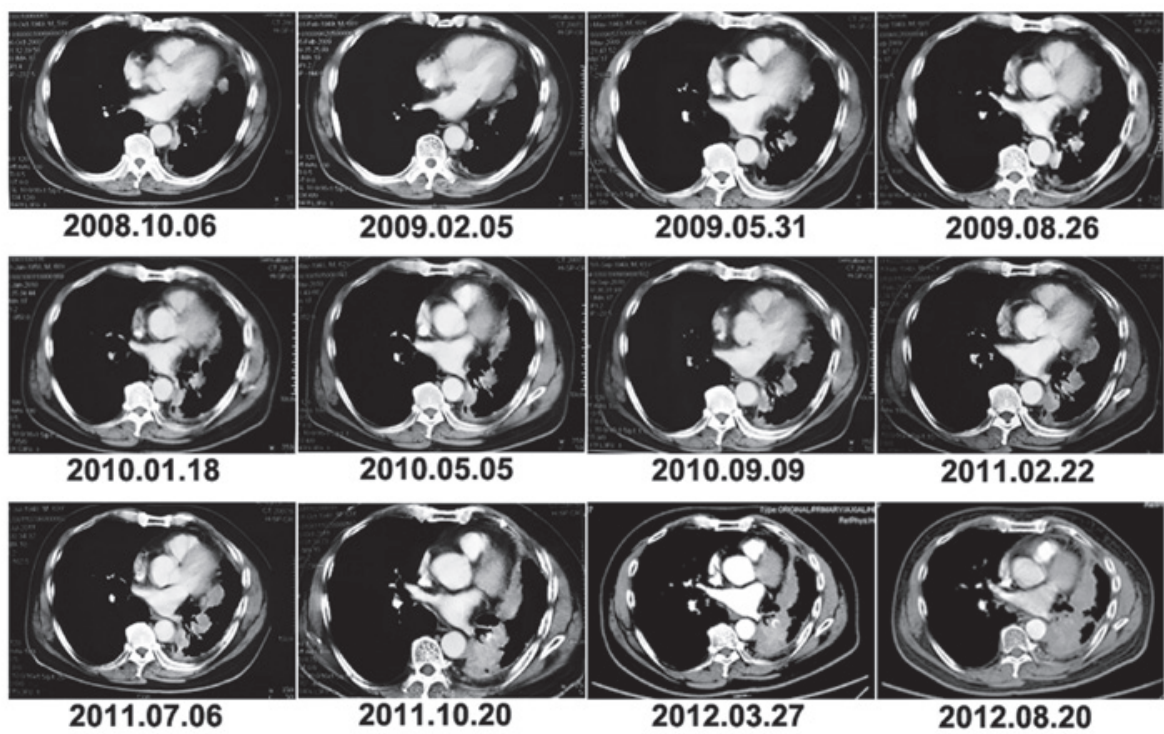

B
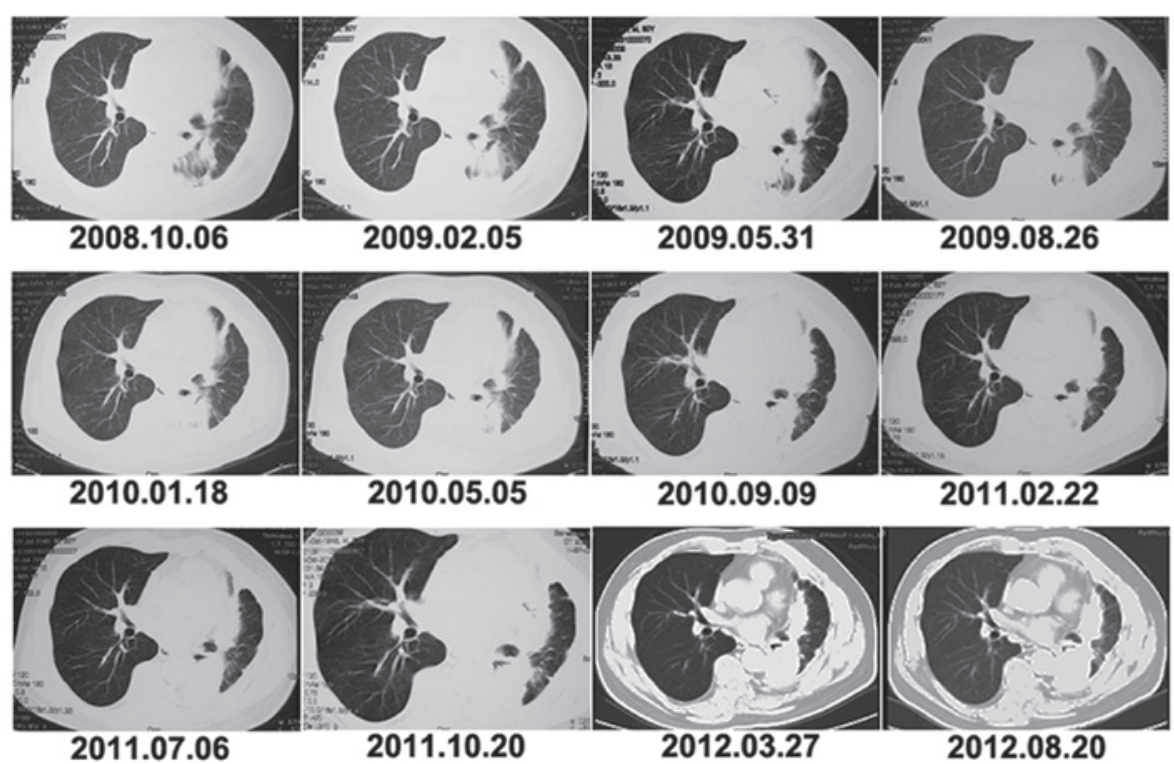

Figure 2. Chest computed tomography images during 24 cycles of maintenance pemetrexed. (A) Mediastinal window and (B) lung window. 


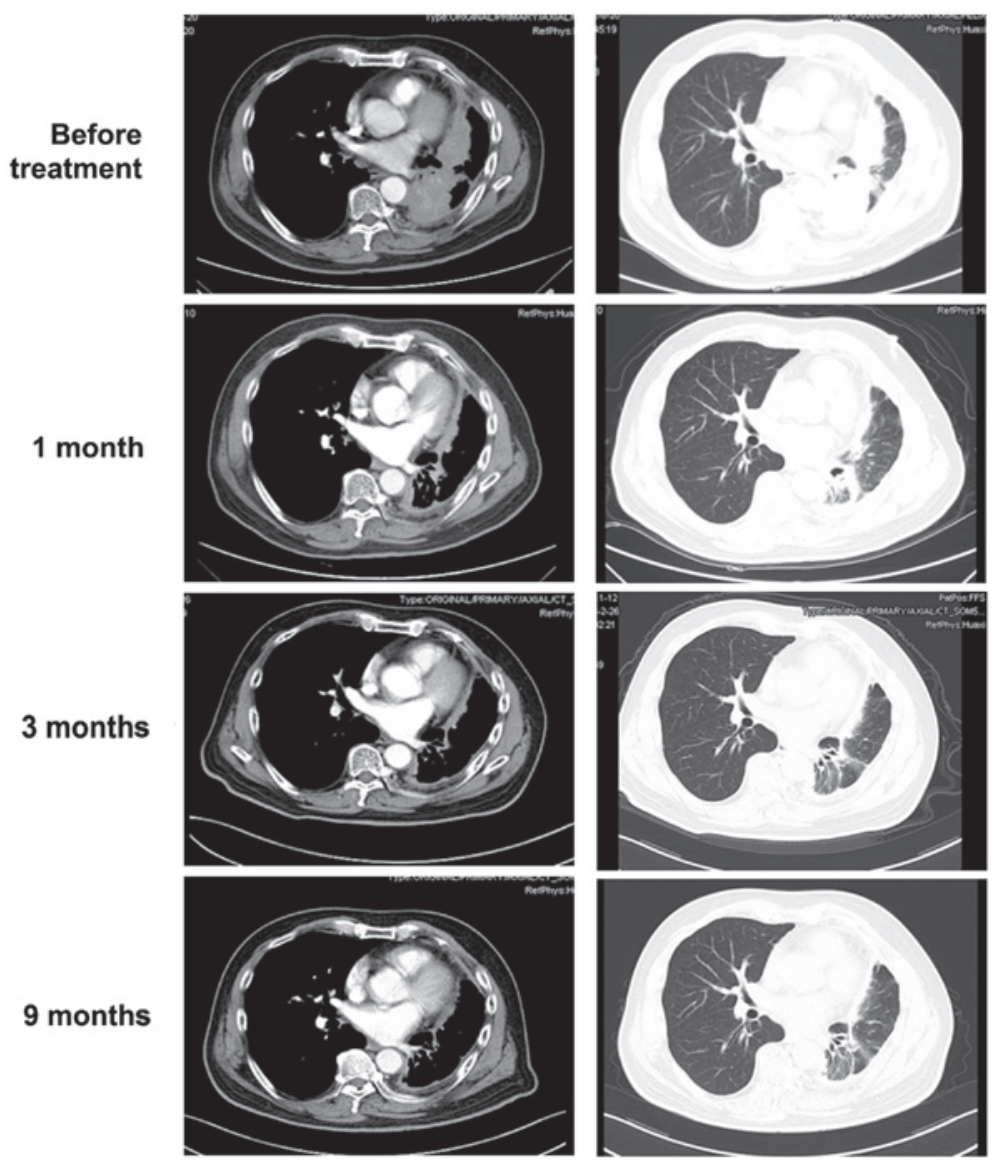

Figure 3. Chest computed tomography before and at 1,3 and 9 months after crizotinib initiation. Left, mediastinal window. Right, lung window.

In May, 2008, the patient was initiated on 4 cycles of pemetrexed and oxaliplatin chemotherapy (Fig. 1B and C), followed by maintenance pemetrexed (900 $\mathrm{mg}$ per cycle). Imaging for response and progression was performed every 2 cycles, with the baseline scans performed after the completion of induction treatment. In May, 2010, after 13 cycles of maintenance pemetrexed, the tumor response was disease progression without any associated symptoms. After consulting with the patient, we continued the administration of pemetrexed and monitored the patient for the development of obvious clinical symptoms or a rapid acceleration of disease progression over a short period of time. The total number of maintenance cycles delivered was 24 (Fig. 2). The patient suffered from grade 1 fatigue, skin pigmentation and periodic fever and requested an increase in the treatment interval. In August, 2012, the patient presented with severe cough and dyspnea following physical activity and the CT images revealed left pleural thickening and an enhancing mass in left lower pulmonary lobe. Rebiopsy was performed with a bronchofiberscope and the histological examination confirmed the diagnosis of adenocarcinoma. The molecular analysis revealed wild-type epidermal growth factor receptor (EGFR) gene and absence of K-ras mutation. However, fluorescence in situ hybridization revealed a translocation of ALK. In November, 2012, oral crizotinib $(250 \mathrm{mg}$ twice per day) was initiated. Transient mild diarrhea and visual disturbances were observed for the first 2 weeks, but were well controlled in the subsequent course of treatment. After 1 month, a chest $\mathrm{CT}$ revealed a significant decrease in the maximum aggregate tumor measurement (Fig. 3). The tumor response according to the RECIST guidelines was partial response. At 52 weeks after the initiation of crizotinib, the patient remained alive and is in confirmed partial response.

\section{Discussion}

Continuation maintenance with pemetrexed is an effective and well tolerated treatment choice for patients with responsive or stable disease after first-line systematic therapy for advanced non-squamous NSCLC $(3,4)$. In this case, first- and second-line therapy failed, mainly due to the side effects; subsequently, the patient entered an induction phase which consisted of 4 cycles of induction pemetrexed plus oxaliplatin. The patient did not exhibit disease progression following completion of the 4 cycles of induction and received maintenance therapy with pemetrexed (900 mg per cycle). According to the RECIST guidelines, the maintenance therapy should be discontinued after 13 cycles of maintenance pemetrexed. As an optimal strategy has not been established for patients receiving third-line therapy and the patient had a good quality of life and exhibited a relatively slow progression on CT imaging, we continued the maintenance therapy, after consulting with the patient, until the development of obvious symptoms. Surprisingly, the patient completed 24 cycles of maintenance pemetrexed.

It was previously reported that ALK-positive NSCLC patients achieved a prolongation of progression-free survival 
on pemetrexed (7). A previous study reported that an ALK-positive patient received 4 cycles of chemotherapy with pemetrexed and carboplatin followed by maintenance pemetrexed therapy for 19.3 months until disease progression (8). The underlying mechanism has not been clearly determined, but it was recently demonstrated that certain enzymes, which catalyze purine biosynthesis, are increased by ALK-mediated phosphorylation. Therefore, ALK-positive tumors may be more susceptible to antifolate agents (9).

Approximately $5 \%$ of the malignant transformation in NSCLC is caused by EML4-ALK rearrangement. Tumor cells with EML4-ALK rearrangement are dependent on its function, similar to tumor cells harboring EGFR mutations. Previous studies reported that crizotinib prolonged progression-free survival, increased response rates and improved the quality of life in patients with advanced ALK-positive NSCLC $(10,11)$. In this case, following a rapid acceleration of disease progression, oral crizotinib (250 mg twice per day) was initiated. The tumor response was remarkable and rapid and the chest $\mathrm{CT}$ revealed a significant decrease in the maximum aggregate tumor measurement within 1 month. The patient experienced transient mild diarrhea and visual disturbances, which were well controlled in the subsequent course of treatment.

Resistance to crizotinib has also been reported. An alteration at C1156 of ALK may allosterically interfere with the binding of tyrosine kinase inhibitors (12). The patient's disease status will be closely followed and further investigations should focus on different treatment strategies using second-generation ALK inhibitors.

\section{References}

1. Siegel R, Naishadham D and Jemal A: Cancer statistics, 2013. CA Cancer J Clin 63: 11-30, 2013.
2. Govindan R, Page N, Morgensztern D, et al: Changing epidemiology of small-cell lung cancer in the United States over the last 30 years: analysis of the surveillance, epidemiologic, and end results database. J Clin Oncol 24: 4539-4544, 2006.

3. Ciuleanu T, Brodowicz T, Zielinski C, et al: Maintenance pemetrexed plus best supportive care versus placebo plus best supportive care for non-small-cell lung cancer: a randomised, double-blind, phase 3 study. Lancet 374: 1432-1440, 2009.

4. Paz-Ares L, de Marinis F, Dediu M, et al: Maintenance therapy with pemetrexed plus best supportive care versus placebo plus best supportive care after induction therapy with pemetrexed plus cisplatin for advanced non-squamous non-small-cell lung cancer (PARAMOUNT): a double-blind, phase 3, randomised controlled trial. Lancet Oncol 13: 247-255, 2012.

5. Shaw AT, Kim DW, Nakagawa K, et al: Crizotinib versus chemotherapy in advanced ALK-positive lung cancer. N Engl J Med 368: 2385-2394, 2013.

6. Kim SJ, Kim DW, Kim TM, et al: Remarkable tumor response to crizotinib in a 14-year-old girl with ALK-positive non-small-cell lung cancer. J Clin Oncol 3: e147-e150, 2012.

7. Camidge DR, Kono SA, Lu X, et al: Anaplastic lymphoma kinase gene rearrangements in non-small cell lung cancer are associated with prolonged progression-free survival on pemetrexed. J Thorac Oncol 6: 774-780, 2011.

8. Takeda M, Okamoto I, Sakai K, et al: Clinical outcome for EML4-ALK-positive patients with advanced non-small-cell lung cancer treated with first-line platinum-based chemotherapy. Ann Oncol 23: 2931-2936, 2012.

9. Boccalatte FE, Voena C, Riganti C, et al: The enzymatic activity of 5-aminoimidazole-4-carboxamide ribonucleotide formyltransferase/IMP cyclohydrolase is enhanced by NPM-ALK: new insights in ALK-mediated pathogenesis and the treatment of ALCL. Blood 113: 2776-2790, 2009.

10. Kijima T, Takeuchi K, Tetsumoto S, et al: Favorable response to crizotinib in three patients with echinoderm microtubule-associated protein-like 4-anaplastic lymphoma kinase fusion-type oncogene-positive non-small cell lung cancer. Cancer Sci 102: 1602-1604, 2011.

11. Shaw AT, Yeap BY, Solomon BJ, et al: Effect of crizotinib on overall survival in patients with advanced non-small-cell lung cancer harbouring ALK gene rearrangement: a retrospective analysis. Lancet Oncol 12: 1004-1012, 2011.

12. Choi YL, Soda M, Yamashita Y, et al: EML4-ALK mutations in lung cancer that confer resistance to ALK inhibitors. N Engl J Med 363: 1734-1739, 2010. 